\title{
Evolution of an Unstable Dynamical System in Mathematical Models of the Theory of Populations of Families of Small Bodies
}

\author{
Gasanbek Arazov", Terane Aliyeva ${ }^{2}$ \\ ${ }^{1}$ Department of Mathematical Modelling and Automated Systems, Institute of Applied Mathematics of Baku State University, Baku, \\ Azerbaijan \\ ${ }^{2}$ Department of Theoretical Physics, Institute of Physical Problems of Baku State University, Baku, Azerbaijan
}

Email address:

arazov_h@yahoo.com (G. Arazov)

To cite this article:

Gasanbek Arazov, Terane Aliyeva. Evolution of an Unstable Dynamical System in Mathematical Models of the Theory of Populations of Families of Small Bodies. World Journal of Applied Physics. Vol. 3, No. 3, 2018, pp. 51-53. doi: 10.11648/j.wjap.20180303.11

Received: October 30, 2018; Accepted: December 8, 2018; Published: January 17, 2019

\begin{abstract}
The sum of an infinite number of forces acts in all points of the space of a dynamical system. The character of this sum of forces corresponds to the characteristic indicators of a dynamic system. Changes in this sum of forces over time lead to the evolution of the system. It may be in stable or unstable states. Unstable systems collapse over time. Their mass and energy are captured by stable systems, as a result of which the characteristic indicators of stable systems also change: they also become unstable and collapse. This process continues until the formation of a single (Main) dynamic system. After formation of the main dynamic system, the whole process is repeated again and again cyclically. Changes in the parameters and composition of matter of the Main Dynamic System, with specially selected initial conditions (as in the evolution of the observed Universe), coincide with changes in the parameters of our Universe in mathematical models of the theory of populations of families of small bodies.
\end{abstract}

Keywords: Automated Dynamic Systems, Evolution, Instability

\section{Introduction}

There are numerous problems in nature whose solutions defy our efforts. In other words, their place, strength (power) and moments of birth cannot be determined and predicted. In the geocentric and heliocentric dynamical systems, many atmospheric and oceanic phenomena, volcanic eruptions, earthquakes, and so on are the examples of such abnormal problems that difficult to explain. This is a consequence of the fact that all natural phenomena and processes are formed and occur under the influence of sums of many large actions as well as sums of infinitely small actions. Moreover, the sum of infinitesimal actions in all processes, it takes part, is hidden. Infinitesimal actions are elusive.

\section{Evolution of Unstable Dynamical System in the Theory of Populations of Families of Small Bodies}

Under special initial conditions, the sum of infinitely small actions can cause a multitude of chaos, catastrophes and other anomalous phenomena that are difficult to explain. The forces of interaction and bombardment control the population in the families of small bodies and they also control the laws of evolution of changes in mass and energy processes.

All points of the sphere of influence of the space of a dynamic system are influenced by the action of the sum of forces: $F+R+\Delta=K$. Mathematical models of the theory of populations of families of small bodies are built on the basis of these quantities. $F=\sum_{i=1}^{n} F_{i}-$ sum of the forces that can be detected by measuring instruments and can be accounted for 
in mathematical simulations. $R=\sum_{j=1}^{\infty} R_{j}-$ sum of the forces that are less than or equal to the errors of the measuring instruments. $\mathrm{R}$ and $\mathrm{F}$ take part in all natural processes and phenomena. $\Delta=\sum_{i=1}^{n} \Delta_{i}-$ sum of the errors of measuring devices. $R=\sum_{i=1}^{n} R_{i}-$ can be determined from equality $R=K-(F+\Delta)$.

As it is shown, "the formation and evolution of the dynamic system is the result of population of stable and unstable orbits of primary fragment -clots- oddments of relict substances and their families, with special initial conditions. Each fragment-clot-oddment of the relict substance has their original chemical composition and primary physical structure. They define their place in space, as well as the stability or instability of their orbits, which became the foundation and the building material of formation of the Sun and planets. The forces of attraction and bombardment by small bodies govern the laws of the evolution of increase of mass and internal energy processes. As a result, the fragments with stable orbits eventually become massive at the expense of fragments with unstable orbits. Areas of stable and unstable movements and formations, i.e. regional populations of celestial bodies, have been discovered in the solar system. As a result of this, the currently observed system appeared. This process continues today in the form of the bombardment by small bodies and their families of the Sun and the planets, including the Earth. $[2]$ "

This paper is a continuation of article [2]. During the decay of unstable dynamic systems, the parameters of some stable systems undergo strong changes. They become unstable and upon reaching certain boundary values (conditions) they break up. This process continues until the formation of a single (Main) dynamic system. Its parameters at zero time moment can be compared with those of the beginning of the universe, i.e. temperature $T=\infty$, pressure $P=\infty$ and mass $M=\infty$. The collapse and the formation of new dynamic systems begin after this.

Table 1. Changes in the parameters and composition of the substance of the Main Dynamic System in mathematical models of the theory of populations of families of small bodies.

\begin{tabular}{|c|c|c|}
\hline \multicolumn{2}{|c|}{ Age of the Main dynamic system } & \multirow{3}{*}{$\begin{array}{l}\text { Temperature } \mathbf{K} \\
n, p, e^{-}, e^{+}, n^{-} \text {in thermal equilibrium } n \text { and } p \text { in the same amount } \\
\text { the same particles but with ratio } n: p=3: 5\end{array}$} \\
\hline $10^{-2} s$ & $10^{11}$ & \\
\hline $10^{-1} s$ & $3 \cdot 10^{10}$ & \\
\hline $1 s$ & $10^{10}$ & $n$ and $n^{-}$are separated from other particles; $e^{-}, e^{+}$begin to void $n: p=1: 3$ \\
\hline $13.8 s$ & $3 \cdot 10^{9}$ & formation of $D$ and ${ }^{4} H e$ begin; $e^{-}, e^{+}$disappear, there are also free $n$ and $p$ \\
\hline $35 \mathrm{~min}$ & $3 \cdot 10^{8}$ & $\begin{array}{l}\text { the amount of } \mathrm{D} \text { и }{ }^{4} \mathrm{He} \text { is being fixed in relation to the number of protons and electrons }{ }^{4} \mathrm{He} / \mathrm{H} \sim \\
22-28 \% \text { (by weight) }\end{array}$ \\
\hline $7 \cdot 10^{5}$ years & $3 \cdot 10^{3}$ & $\begin{array}{l}\text { Binding chemical energy becomes residual to form stable neutral atoms. Now the main dynamic system is } \\
\text { transparent to radiation. Substance begins to dominate under radiation. }\end{array}$ \\
\hline
\end{tabular}

Designations in the table: $n$ - neutron, $p$ - proton, $e^{-}$- electron, $e^{+}-$positron, $n^{-}-$neutrino, $n^{+}-$antineutrino, $D-$ deuterium, He- helium.

It is noted that only a certain set of parameters correspond to all dynamic systems observed in nature. In mathematical modelling, the choice of a parameter set is arbitrary. The arbitrariness of the choice of parameters allows a comparative analysis of various dynamic processes and systems. The geocentric and heliocentric dynamical systems are well-studied parts of the Universe. And the Universe is one of the possible variants of a single (Main) dynamic system. Therefore, with an appropriate choice of initial data (conditions) of a single (Main) dynamic system [2], changes in one of the possible variants of this system can be presented in the form of table 1 .

\section{Conclusion}

Thus, the formation and evolution of a dynamic system can occur in accordance with well-defined laws (rules). They are based on a population of stable and unstable orbits of primary fragments of relict substances and their families with special initial conditions. Each fragment-clot of relict substance has its original composition and primary physical fractures. They were the beginning of regional processes of a population of particle families and small bodies. As an example, the equivalence of changes in the parameters and composition of the Main Dynamic System with those observed in the Universe is considered.

\section{References}

[1] Carey S. U., 1991. In search of the laws of development of the Earth and Universe, Moskva, Peace, 447 p.

[2] Arazov G. T., Aliyeva T. H. Formation and Evolution of Sustainable Dynamic System Mathematical Models of the Theory of Population of Families of Small Bodies. Modern Thrends in physics. Conference Proceedings International Conference. Baku, 2017, page 135-137.

[3] Cherniy A. N., From the Big Bang to the accelerated expansion of the Universe. News of Educational Institutions: Astronomy and Space Geodesy. Academy of Electrical Engineering of the Russian Federation. Moscow, volume 60, № 4, 2016, page 3-7. 
[4] Abraham Loeb and Steven R. Furlanetto. 2013. The first galaxies in the Universe, Prinston University Press. Prinston and Oxford, page 540.

[5] Oded Regev. 2006. Chaos and complexity in Astrophysics, Cambridge University Press, page 455.

[6] Safronov V. S., 1969, The evolution of the protoplanetary cloud and Earth and planetary formation, Moscow, Science, page 347.
[7] Sun Y. S., Zhou L. Y., 2016. From ordered to chaotic motion in Celestial Mechanics, Nanjing University, China, World Scientific, page 405 .

[8] Zeleniy L. M., Zakharov A. V., Ksanfomaliti L. V., 2009. Researches of the solar system, the state and prospects, Moscow, Science, Russian Academy of Sciences, Volume 179, № 10, page 1118-1140. 